Conclusion This pilot study has shown the DCS could be a useful tool for the prediction of difficult colonoscopy. This could be of benefit when scheduling lists for training and choosing the level of experience of colonoscopists before procedures are performed. A large study is planned.

Competing interests None declared.

\section{PTU-229 THE EFFECT OF FRAME RATE AND VIEW MODE ON LESION DETECTABILITY BY NOVICE AND EXPERT CAPSULE ENDOSCOPISTS DURING READING}

doi:10.1136/gutjnl-2012-302514c.229

M Nakamura, ${ }^{*}$ A Murino, A Fitzpatrick, Y Komeda, R La Nauze, S Green, C Fraser. The Wolfson Unit for Endoscopy, St Mark's Hospital and Academic Institute, Imperial College, London, UK

Introduction The RAPID 7 Access reading software (Given Imaging Ltd) allows the capsule endoscopist to adjust the frame rate of presented images (adjustable frame rate, AFR) and their view mode (VM1 - single view; VM2 - dual view; VM4 - quad view) during capsule endoscopy (CE) reading. The aim of this study was to establish the relationship between AFR, VM, lesion miss rate and reading time between non-expert (NEXs) and expert (EXs) capsule endoscopists.

Methods One short video clip containing 60 positive images of angioectasias was selected from our CE database. The clip was read by 3 EXs and 3 NEXs using nine different combinations of VM and $\operatorname{AFR}(1,2$ and $4 \mathrm{VMs} \times 10,15$ and $25 \mathrm{fps}$ ) presented in randomised order. Readers were asked to count each positive image of an angioectasia using a manual counter, without interrupting the video clip.

Results The reading times at 10, 15 and 25 fps were 54, 34 and 20 s, respectively for any VM. Considering $10 \mathrm{fps}$ as the gold standard, an AFR of 15 and $25 \mathrm{fps}$ resulted in a reduction in reading time of $37 \%$ and $63 \%$ respectively. The number of positive images detected using 10, 15 and $25 \mathrm{fps}$ (all VM's combined) were 45, 31 and 22 respectively. The mean number of detected positive images (MPI) using $10 \mathrm{fps}$ was significantly higher than an AFR of 15 and $25 \mathrm{fps}$ $(\mathrm{p}=0.04,0.01)$. For VM1, VM2 and VM4, the MPI was 24,36 and 38 respectively. The MPI using VM2 and VM4 was significantly higher than for VM1 $(p=0.01,0.003)$. VM4 $\times 10 \mathrm{fps}$ had highest MPI (51) while VM1 $\times 25$ fps had the lowest MPI (14). MPIs of NEX and EX (all VM's combined) were 34 and 32 and were not significantly different.

Conclusion While a higher AFR results in a reduction in reading time, lesion detectability is reduced and miss rates increase. Higher MPIs are associated with lower AFRs and an increase in VM. In this study the optimal combination for lesion detectability was VM4 $\times$ $10 \mathrm{fps}$. NEXs and EXs performed similarly for the detection of angioectasias.

Competing interests None declared.

\section{PTU-230 OUTCOMES OF ENDOSCOPIC HUMAN THROMBIN INJECTION IN THE MANAGEMENT OF GASTRIC VARICES}

doi:10.1136/gutjnl-2012-302514c.230

M Smith, ${ }^{*}$ R Tidswell, D Tripathi. Liver Unit, University Hospitals Birmingham NHS Trust, Birmingham, UK

Introduction The optimal therapy for gastric variceal bleeding remains unclear. Endoscopic Human Thrombin injection appears a technically simple and efficacious alternative to cyanoacrylate with fewer complications, but data remains limited. This study evaluated patient outcomes following thrombin injection for gastric varices. Methods Retrospective review of patients receiving endoscopic human thrombin injection for active bleeding or prevention of bleeding from gastric varices at a UK tertiary centre from December 2008 to November 2011. Thrombin injection (Tisseel $250 \mathrm{IU} / \mathrm{ml}$, Baxter Int. Inc.) was repeated at intervals until varices eradicated.

Results 23 patients ( $65 \%$ male, mean age 53.1 (SD 14.0)), received human thrombin injection for gastric varices. Mechanism of portal hypertension was cirrhosis 17 patients (74\%), extra-hepatic $6(26 \%)$. Cirrhosis was due to alcohol (10), viral (2), PBC (2), other (3); 4 had additional portal vein thrombosis. TIPSS was felt not feasible in 8 (35\%). Mean MELD was 13 (SD 5). Childs grade A, B, C in 39\%, 35\% and $26 \%$ respectively. Varices were classified: IGV1 19 (83\%), IGV2 3 (13\%), GOV2 1 (4\%). 14 patients (61\%) were actively bleeding or had signs of recent bleeding; of these haemostasis was achieved in 12 (86\%). Mean thrombin dose/injection was $1168 \mathrm{IU}$ (range 400-2500); median number of sessions 2 (range 1-7) with no reported complications. Median follow-up was 476 days (IOR 193-931). No patient underwent liver transplantation. Rebleeding occurred in $9(39 \%)$ patients, $5(56 \%)$ within the first week (range $1-1008$ days), 1 yr rebleeding rate $35 \%$. Rebleeding was successfully managed in $78 \%$, by salvage TIPSS (5 patients) and thrombin injection (2). Two patients died following rebleeding. Six deaths (26\%) occurred in total all within 12 months; the remainder were due to uncontrolled bleeding (1), liver failure (1), MOF following OV bleed banded (1), and hepatocellular carcinoma (1). Cumulative survival at 1, 6, 12 months was $82 \%, 78 \%$, and $74 \%$ respectively. Where TIPSS was precluded, $75 \%$ ( 6 of 8 patients) were managed successfully with thrombin.

Conclusion Thrombin in our series appears to be a safe and effective endoscopic therapy for gastric varices, achieving good haemostasis with low medium to long term rebleeding rates. It may have particular utility in salvaging patients not suitable for TIPSS.

Competing interests None declared.

\section{PTU-231 BOWEL PREPARATION FOR INPATIENT COLONOSCOPY: AN AUDIT OF OUALITY AND OUTCOMES}

doi:10.1136/gutjnl-2012-302514c.231

M Aldridge, ${ }^{*}$ A Phillips, I Gee. Department of Gastroenterology, Worcestershire Acute Hospitals NHS Trust, Worcester, UK

Introduction It is well recognised that inpatient colonoscopy is more problematic than outpatient colonoscopy, with poorer quality of bowel preparation ${ }^{1}$ and reduced rates of successful completion of the procedure among inpatients. ${ }^{2}$ We aimed to measure the quality of bowel preparation and the success rate of inpatient colonoscopy in a large district general hospital.

Methods All patients undergoing inpatient colonoscopy at Worcestershire Royal Hospital between 1 September 2010 and 1 September 2011 were identified retrospectively using paper-based documentation available in the Endoscopy department. The computerised colonoscopy reports (Unisoft, Enfield, UK) were then obtained for these patients. Standard bowel preparation for these patients was two sachets of Picolax, one the evening before and one the following morning, with colonoscopy performed on an afternoon list. Successful colonoscopy was defined as intubation of the caecum with "excellent" or "good" bowel preparation.

Results We identified 50 patients undergoing inpatient colonoscopy, with a median age of 74 (IOR 62-80), representing 3\% of all colonoscopies done during this period. Approximately one-third (38\%) were performed due to suspicious symptoms (most commonly PR bleeding), one-third (34\%) were performed due to a CT abnormality, 
with the remainder predominantly due to unexplained anaemia (18\%). Only 50\% (25/50) of inpatient bowel preparation was rated by the endoscopist as "excellent" or "good," compared with $86 \%$ overall for the same period ( $p<0.001$ by $\chi^{2}$ analysis). Among endoscopists with individual overall caecal intubation rates of $>90 \%$, the inpatient caecal intubation rate was only $74 \%(37 / 50)$. Out of the 13 failed inpatient intubations, 7 (54\%) were due to poor bowel preparation. The remainder were due to patient discomfort (3), difficult angulation (2), and malignancy (1). In addition, the overall inpatient success rate was only $66 \%(33 / 50)$. In four cases (8\%), although caecal intubation was achieved, poor bowel preparation meant a small lesion could not be excluded.

Conclusion This audit has demonstrated that the failure rate for inpatient colonoscopy is greater than outpatient procedures. The majority of these failures are due to poor bowel preparation. The reasons for this are complex, but may include reduced mobility and poorer adherence to bowel preparation and oral hydration. Deferring colonoscopy until after discharge from hospital is therefore advised whenever possible.

Competing interests None declared.

\section{REFERENCES}

1. Ness RM, Manam R, Hoen $\mathrm{H}$, et al. Predictors of inadequate bowel preparation for colonoscopy. Am J Gastroenterol 2001;96:1797-802.

2. Hendry PO, Jenkins JT, Diament RH. The impact of poor bowel preparation on colonoscopy: a prospective single centre study of 10,571 colonoscopies. Colorectal Dis 2007:9:745-8.

\section{PTU-232 EVALUATING THE ROLE OF CAPSULE ENDOSCOPY IN EOUIVOCAL COELIAC DISEASE?}

doi:10.1136/gutjnl-2012-302514c.232

M Kurien, ${ }^{*}$ K E Evans, R Sidhu, K Drew, M E McAlindon, D S Sanders. Department of Gastroenterology, Royal Hallamshire Hospital, Sheffield, UK

Introduction Demonstration of villous atrophy (VA) on small bowel biopsy and positive serology (endomysial antibody (EMA) and/or tissue transglutaminase (tTG)) is the current gold standard for diagnosing coeliac disease. Difficulty in establishing the diagnosis may arise for several reasons. A minority may have antibody negative disease. Some individuals may have positive antibodies with histological changes that fall short of VA (Marsh Grade 1 and 2 [MG1-2]) or are unable to tolerate gastroscopy. In addition, not all VA seen is caused by coeliac disease. The aim of this study was to assess the value of capsule endoscopy (CE) in equivocal coeliac disease.

Methods Data from all patients with equivocal coeliac disease who underwent CE between 2004 and 2011 in a tertiary gastroenterology department were analysed. Patients were subdivided into five main groups: Group 1-antibody negative VA; Group 2-MG1-2; Group 3 - positive coeliac serology with normal duodenal biopsy; Group 4-miscellaneous including strong family history and non-gastrointestinal presentation of probable coeliac disease; Group 5-failed or refused gastroscopy. Demographic data, indication for $\mathrm{CE}$, serology and histology were recorded prospectively. Videos were analysed by two experienced gastroenterologists blinded to the clinical data. Markers of coeliac disease such as scalloping, mosaic pattern and loss of folds were assessed. A diagnosis of coeliac disease was further supported by not only CE appearances but also combinations of HLA typing (DQ-2 or DQ-8), gluten challenge/ response to a gluten free diet and in some cases repeat duodenal biopsy.

Results 102 patients, 72 female, median age 49 years, (range $18-89$ y) underwent CE. 17/102 (16\%) had features of coeliac disease on CE, with a further three cases of Crohn's disease identified (Abstract PTU-232 table 1). In patients with coeliac antibody negative VA, CE secures a diagnosis of coeliac or Crohn's in 9/32 $(28 \%)$ significantly more than in other groups where previous gastroscopy was undertaken $(p=0.04)$. In $57 \%(4 / 7)$ of patients with positive coeliac serology who either failed or refused gastroscopy, CE helped establish the diagnosis.

Abstract PTU-232 Table 1

\begin{tabular}{llll}
\hline & $\begin{array}{l}\text { Normal } \\
\text { CE }(\mathbf{n})\end{array}$ & $\begin{array}{l}\text { Features } \\
\text { of coeliac }\end{array}$ & $\begin{array}{l}\text { Other CE } \\
\text { diagnosis }\end{array}$ \\
\hline Antibody -ve VA $(\mathrm{n}=32)$ & 23 & 7 & 2 Crohn's \\
MG1-2 $(\mathrm{n}=29)$ & 26 & 2 & 1 Crohn's \\
+ve antibody, normal biopsy $(\mathrm{n}=10)$ & 9 & 1 & 0 \\
Miscellaneous ( $\mathrm{n}=24)$ & 21 & 3 & 0 \\
Failed/refused gastroscopy $(\mathrm{n}=7)$ & 3 & 4 & 0 \\
\hline
\end{tabular}

Conclusion CE may have a role in the assessment of patients with coeliac antibody negative VA and in antibody positive patients where previous gastroscopy has been refused or failed. Its routine use is not supported in other causes of "equivocal" coeliac disease.

Competing interests None declared.

\section{PTU-233 IN VIVO POLYP SIZE AND HISTOLOGY ASSESSMENT AT COLONOSCOPY: ARE WE READY TO RESECT AND DISCARD? A MULTI-CENTRE ANALYSIS OF 1212 POLYPECTOMIES}

doi:10.1136/gutjnl-2012-302514c.233

${ }^{1} \mathrm{M} F$ Hale, ${ }^{*} \mathrm{M}$ Kurien, ${ }^{1} \mathrm{P}$ Basumani, ${ }^{3} \mathrm{R}$ Slater, ${ }^{2} \mathrm{D} S$ Sanders, ${ }^{2} \mathrm{~A} D$ Hopper. ${ }^{1}$ Department of Gastroenterology, Rotherham Hospital, Rotherham, UK; ${ }^{2}$ Department of Gastroenterology, Royal Hallamshire Hospital, Sheffield, UK; ${ }^{3}$ Department of Surgery, Rotherham Hospital, Rotherham, UK

Introduction The current paradigm of colonoscopic management of polyps is to resect and send for pathologic assessment. Such practice incurs substantial costs for a group of lesions with limited clinical importance. Several studies have proposed a resect and discard approach for smaller polyps. For this to be effective our in vivo assessment of polyp size needs to be accurate. Additionally a high positive predictive value (PPV) of adenomas among polyps resected is essential to ensure patients are correctly risk stratified for surveillance.

Methods All polypectomies performed from 1 January 2010 to 31 December 2010 were identified retrospectively from databases in a dual site teaching hospital and local district general endoscopy units. Polyps removed and retrieved with corresponding histology were identified. Polyp site, endoscopic size and endoscopist specialty were recorded. Carcinomas, adenomas and serrated lesions were determined to be neoplastic. The total number of neoplasms removed divided by the total number of polyps removed was calculated (PPV). Fishers exact test was used to compare the subspecialties of endoscopist (nursing/surgical/medical). In vivo size was analysed for terminal digit preference by the colonoscopist compared to histology measurements using a $\chi^{2}$ goodness of fit test. Calculations of the distribution of error between in vivo estimation and histology measurement and the number of times the size discrepancy crossed the $10 \mathrm{~mm}$ value used in planning surveillance colonoscopy were performed.

Results 1212 polyps were included, 864 had in vivo size estimation and subsequent en-bloc histology measurements $\leq 20 \mathrm{~mm}$. The PPV for neoplastic polyps was 69\% (831/1212) with 381 non-neoplastic polyps removed. Nurse endoscopists had the highest PPV, 74\% $(\mathrm{n}=347 / 486)$ compared to surgeons (PPV 72\%: 143/199) and medics (PPV 64\%: 339/527, $\mathrm{p}<0.02$ ). Considering proximal hyperplastic polyps as neoplastic the overall PPV $=73 \%$ (879/1212), nurses PPV 